\title{
The Influence of Corneal Volume in Surgical Planning: Insights for a New Parameter for the Ferrara Ring Nomogram
}

\author{
Leonardo Torquetti, Guilherme Ferrara, Wagner Gomes Dias, Paulo Ferrara
}

\begin{abstract}
Purpose: To evaluate the corneal volume (CV) before and after Ferrara intrastromal corneal ring segments (ICRS) implantation and its influence in clinical outcomes in keratoconus patients.

Materials and methods: A total of 77 eyes of 42 keratoconus patients consecutively implanted with the Ferrara ICRS were evaluated with a Pentacam (Oculus Optikgerate $\mathrm{GmbH}$ ). The following parameters were obtained: Anterior corneal asphericity (Q), CV and thinnest corneal thickness (TCT). The inter-relation between $\mathrm{Q}$ and $\mathrm{CV}$ was evaluated.
\end{abstract}

Results: In patients with CV inside two standard deviation (SD: 53.51-60.65) of the mean CV, the postoperative asphericity values roughly agreed with expected asphericity values. In patients with CV outside two SD intervals ( $<53.51$ and $>60.65$ ) of the mean CV, the postoperative asphericity values did not agreed with expected asphericity values. Corneas with high volumes ( $>60.65$ ) had less change of asphericity than corneas with low volumes.

Conclusion: The CV is a new parameter, to be better studied, as it seems to be important in ICRS selection. Corneas with high volumes may require more tissue to get flattened and properly reshaped.

Keywords: Corneal volume, Pentacam, Tomography, Keratoconus.

How to cite this article: Torquetti L, Ferrara G, Dias WG, Ferrara $P$. The Influence of C orneal Volume in S urgical Planning: Insights for a New Parameter for the Ferrara R ing Nomogram. Int J Kerat Ect Cor Dis 2013;2(1):12-15.

\section{Source of support: Nil}

Conflict of interest: None declared

\section{INTRODUCTION}

Keratoconus is a bilateral, progressive noninflammatory corneal ectasia that tends to affect young age groups in their late teens. ${ }^{1}$ Despite intensive clinical and laboratory investigations, its precise etiology is unknown. ${ }^{2,3}$ In keratoconus, corneal changes result in a mild to severe decrease in the corrected distance visual acuity (CDVA) as a result of progressive myopia, regular and irregular astigmatism, in addition to apical scarring in advanced cases. $^{4}$

One of the goals of treatment of keratoconus with intrastromal corneal ring segments (ICRS) is to improve quality of vision beyond the simple corneal flattening and stabilization of the disease. Significant asphericity changes can occur after any corneal surgery ${ }^{5,6}$ and these changes may explain the increase in spherical aberration and deterioration in the quality of monocular and binocular vision. 7,8

The actual Ferrara ICRS nomogram is based on corneal asphericity. ${ }^{9}$ Previously published studies have shown that is possible to predict the final Q-value after ICRS implantation. ${ }^{10}$ This is important, once the quality of postoperative vision is linked to, besides the corneal flattening, the corneal shape (oblate or prolate). It is well know $n$ that not only the excess of prolateness of the cornea found in keratoconus but also the cornea oblate can cause unsatisfactory visual quality. Therefore, the surgical plan should be aimed to achieve a normal, physiologic Q-value $(-0.23 \pm 0.08){ }^{11}$

Corneal volume (CV) was recently identified as an additional screening factor for keratoconus. ${ }^{12-14}$ Significant differences in CV have been reported between normal and moderate keratoconic eyes (Pentacam system: $60.83 \pm 3.27$ $\mathrm{mm}^{3}$ controls vs $57.98 \pm 2.65 \mathrm{~mm}^{3}$ moderate keratoconus), ${ }^{12}$ suggesting the potential role for CV as a diagnostic factor for corneal ectatic disorders. However, there is not enough scientific evidence of the potential usefulness of $\mathrm{CV}$ as a screening factor for keratoconus suspect.

The aim of the present study was to eval uate changes in the $\mathrm{CV}$ and asphericity before and after ICRS implantation and the correlation between these parameters.

\section{MATERIALS AND METHODS}

In the present study, 77 eyes of 42 consecutive surgical patients from J anuary to M arch 2011 were evaluated. The preoperative and postoperative data of patients, which had a Ferrara ICRS implantation, were used for analysis. This study was approved by the institutional review board of Dr Paulo Ferrara E ye Clinic, B elo H orizonte, M inas Gerais, $B$ razil and followed the tenets of the Declaration of $\mathrm{Helsinki.}$ The procedures were fully explained to each patient, and each provided written informed consent.

All operated patients had the diagnosis of keratoconus based on clinical findings and tomographic parameters. The patients had progressive intolerance to contact lens and/or clinical and topographic evidence of disease progression and/or unsatisfactory CDVA. 
The Influence of Corneal Volume in Surgical Planning: Insights for a New Parameter for the Ferrara Ring Nomogram

Participant exclusi on criteria were any previous corneal or ocular surgery, any eye disease other than keratoconus and chronic or continuous use of topical medications. Contact lenses (soft or rigid) had to be removed at least 72 hours before the examination.

We evaluated the asphericity changes after ICRS implantation and the expected asphericity ${ }^{9,10}$ change based on preoperative $\mathrm{CV}$.

\section{PENTACAM MEASUREMENTS}

The Pentacam is a combined device consisting of a slit illumination system and a Scheimpflug camera, which rotates around the eye.

A thin layer within the eye is illuminated through the slit. B eing not entirely transparent the cells scatter the slit light. In doing so, they create a sectional image, which is then photographed in side view by a camera. This camera is oriented according to the Scheimpflug principle, thus creating an image of the illuminated plane, which appears completely sharp from the anterior surface of the cornea right up to the posterior surface of the crystalline lens.

A fter correction for Scheimpflug distortion and light refraction at tissue interfaces the exact of location of image edge points in the eye is determined by means of raytracing. ${ }^{15}$ Eye movements during image acquisition are captured by a second camera (pupil camera) and also taken into account in the mathematical evaluation. This produces a set of three-dimensional (3D) measurement data, which gives a precise geometric description of the anterior eye segment. This data in turn can be used to generate data on elevation, curvature, pachymetry and depth of the anterior chamber in the well known form of color maps. We used the HR version of Pentacam, which has a precision and reproducibility of $0.1 \mathrm{D}$ for keratometry measurements.

The following parameters were evaluated with the Scheimpflug system: M ean corneal power in the $3.0 \mathrm{~mm}$ zone, topographic astigmatism, CV at $10 \mathrm{~mm}$, minimum pachymetry and mean asphericity at $4.5 \mathrm{~mm}$ diameter corneal area.

\section{STATISTICAL ANALYSIS}

All data were analyzed using the SPSS software (SPSS, Chicago, IL) and reported as means \pm standard deviation. The Student's t-test for paired sample was used for comparison of the variables. A p-value less than 0.05 was considered statistically significant.

\section{RESULTS}

The mean preoperative CV was 57.08 (SD: standard deviation \pm 3.57 ) and 58.92 postoperatively (Table 1 ). The
Table 1: Preoperative and postoperative parameters

\begin{tabular}{lcc} 
& Preoperative & Postoperative \\
\hline Q-value & -0.86 & -0.33 \\
Corneal volume & 57.08 & 58.92 \\
Pachymetry (thin) & 454 & 470 \\
Km & 56.3 & 51.4 \\
Top. astigmatism & -2.68 & -1.09 \\
\hline
\end{tabular}

Student's t-test for paired sample, $p<0.001$ for all parameters

keratometry decreased from 56.3 to $51.4 \mathrm{D}(p<0.001)$. The mean asphericity increased from -0.86 to -0.33 ( $p<$ 0.001 ). We evaluated the asphericity changes after ICRS implantation and the expected asphericity change based on preoperative CV. The Table 2 shows, in a sample of patients, the preoperative and postoperative $\mathrm{Q}$ and $\mathrm{CV}$, and the $\Delta \mathrm{Q}$ and expected $\Delta Q$. In patients with CV inside tw o SD (53.5160.65 ) of the mean $C V$, the postoperative asphericity values roughly agreed with expected asphericity values. In patients with CV outside two SD interval $(<53.51$ and $>60.65)$ of the mean CV , the postoperative asphericity values did not agreed with expected asphericity values. Corneas with high volumes (>60.65) had less change of asphericity than corneas with low volumes.

\section{DISCUSSION}

A mong the numerous morphologic parameters that can be measured by modern examination techniques is the $\mathrm{CV}$. It reflects topographical and pachymetric changes and characterizes corneal morphometric changes with a single value. ${ }^{16}$ The Pentacam, a 3D analyzer equipped with a rotating Scheimpflug camera, allows CV assessment. B ecause the CV is a numerical value, it may be useful for a statistical assessment of the entire cornea.

$\mathrm{CV}$ has been proposed as a new index to diagnose keratoconus and screen refractive candidates. ${ }^{12}$ How ever, most published papers found no significant differences in CV among different grades of keratoconus. ${ }^{13,17}$

$M$ annion et al have found that CV was significantly decreased in keratoconus, particularly in the central and paracentral area explained by loss of corneal tissue. The reduction in CV was in moderate and severe cases of keratoconus, but not in the early cases. One likely explanation for this finding could be that in early stages of keratoconus, a redistribution of CV occurs with no loss of tissue. ${ }^{18}$

M ost studies agree that the human cornea Q (asphericity) values ranges from -0.01 to $-0.80 .{ }^{11-13}$ Currently, the most commonly accepted value in a young adult population is approximately $-0.23 \pm 0.08 .{ }^{14} \mathrm{As}$ the asphericity can be considered as one of markers of quality of vision, ${ }_{1}^{15}$ turning it closer to 'normal' or at least reducing the excess of prolateness usually found in keratoconus, could be a 
Table 2: Preoperative and postoperative $\mathrm{Q}$ and $\mathrm{CV}$; achieved and expected

\begin{tabular}{lccccccc}
\hline Patient & Q preop & CV preop & Q postop & $\begin{array}{c}\text { Delta Q } \\
\text { achieved }\end{array}$ & $\begin{array}{c}\text { Delta Q } \\
\text { expected }\end{array}$ & CV postop & Ring used \\
\hline ARF & -1.21 & 63.7 & -1.19 & -0.02 & -1.02 & 64.3 & $160 / 250$ and $160 / 200$ \\
BGFG & -0.51 & 52.4 & -0.1 & -0.41 & -0.26 & 54.3 & $160 / 150$ \\
CMFT & -0.87 & 64.8 & -0.95 & 0.08 & -0.34 & 64.8 & $160 / 250$ \\
EB & 0.34 & 60.6 & 0.45 & -0.11 & -0.31 & 59.3 & $140 / 150$ \\
LMA & -1.85 & 61.6 & -1.61 & -0.24 & -0.86 & 68.8 & $160 / 200 \times 2$ \\
LRJ & -0.61 & 52.3 & -0.17 & -0.44 & -0.26 & 54.1 & $160 / 150$ \\
MFAS & -0.85 & 62.1 & 0.25 & -1.1 & -0.57 & 63.7 & $160 / 150$ \\
MFAS & -0.64 & 61.8 & 0.18 & -0.82 & -0.57 & 63.4 & $160 / 15 \times 20$ \\
MLOF & -0.28 & 50 & 0.02 & -0.3 & -0.26 & 50 & $160 / 150$ \\
RMM & -1.15 & 51.9 & -0.36 & -0.79 & -0.73 & 52.7 & $160 / 200$ and $160 / 150$ \\
RM & -1.1 & 62.5 & 0.7 & -1.8 & -0.8 & 64.1 & $160 / 250$ and $160 / 150$ \\
SDMS & 0.02 & 51.4 & -0.05 & 0.07 & -0.26 & 54.3 & $160 / 150$ \\
THQV & -1.71 & 51.9 & -0.61 & -1.1 & -0.86 & 53 & $160 / 200 \times 2$ \\
VRC & 0.03 & 61.1 & -0.26 & 0.29 & -0.86 & 61.8 & $160 / 150$ \\
\hline
\end{tabular}

predictor of improvement of visual. The Q-value has been used as an important parameter for ICRS selection. ${ }^{16,17}$

A recently published paper has shown how much each thickness of ICRS (single or paired) can change the cornea asphericity. ${ }^{10}$ Based on this data we can expect the asphericity change based on the thickness of the segment implanted. In the present paper we evaluated the asphericity change and the expected asphericity change after ICRS implantation and correlated this data with the preoperative $\mathrm{CV}$ in order to evaluate its influence in the outcomes based on $\mathrm{Q}$.

We found that in patients with the CV inside two SD (53.51-60.65) of the mean CV , the postoperative asphericity values roughly agreed with expected asphericity values. In patients with CV outside two SD intervals $(<53.51$ and $>60.65$ ) of the mean $C V$, the postoperative asphericity values did not agreed with expected asphericity values. M oreover, patients with cornea volume higher than 60.65 may have worse outcomes based on corneal asphericity.

Tu et al ${ }^{19}$ implanted symmetrical segments of the same thickness in a sample of keratoconus patients with different grades of the disease. They reported that the refractive and keratometric surgical effect of ICRS appeared to be positively associated with the stage of keratoconus; that is, the effect was greater the more advanced the keratoconus stage. Considering that the $\mathrm{CV}$ is lower in more advanced cases of keratoconus, the results of that study agrees with the results of the present paper.

A mbrosio et al ${ }^{12}$ showed that the corneal-thickness spatial profile, corneal-volume distribution, percentage of increase in thickness, and percentage of increase in volume are different in keratoconic eyes and normal eyes. $K$ eratoconic eyes have thinner corneas than normal corneas, with less volume and a more abrupt increase in these parameters from the thinnest point toward the periphery. In that paper, the authors described the corneal thickness and volume in different optical zones.
The CV is a new parameter, to be better studied, as it seems to be important in ICRS selection. Corneas with high volumes may require more tissue to get flattened and properly reshaped. The presented data could be used, in future studies, to aid in the development of new nomograms for ICRS implantation.

\section{REFERENCES}

1. Duke-Elder S, Leigh A G. System of ophthalmology. Diseases of the outer eye. London: Henry Kimpton 1965;964-76.

2. K emp EG, Lewis EJ. Immunoglobulin patterns in keratoconus with particular reference to total and specific IgE levels. B $\mathrm{r}$ J Ophthalmol 1982;66(11):717-20.

3. K arseras A G, Ruben M. A etiology of keratoconus. $\mathrm{Br}$ J Ophthalmol 1976;60(7):522-25.

4. Tomidokoro A, Oshika T, A mano S, Higaki S, Naoyuki M, $K$ azunori $M$. Changes in anterior and posterior corneal curvatures in keratoconus. Ophthalmology 2000;107(7):1328-32.

5. A nera $R G$, J iménez J $R$, Jiménez del $B$ arco $L$, et al. Changes in corneal asphericity after laser in situ keratomileusis. J Cataract Refract Surg 2003;29:762-68.

6. Jiménez JR, A nera RG, Díaz JA, Pérez-Ócon F. Corneal asphericity after refractive surgery when the $M$ unnerlyn formula is applied. J O pt Soc A m A Opt Image Sci V is 2004;21:98-103.

7. Jiménez JR, A nera RG, Jiménez del $B$ arco $L$. Equation for corneal asphericity after corneal refractive surgery. J Refract Surg 2003;19:65-69.

8. J iménez Cuesta JR, A nera RG, Jiménez R, Salas $C$. Impact of interocular differences in corneal asphericity on binocular summation. A m J O phthalmol 2003;135:279-84.

9. Ferrara $P$, Torquetti $L$. The new Ferrara ring nomogram: The importance of corneal asphericity in ring selection. Vision Panamerica, September 2010. A vailable from: http:// www.paao.org/pdf/vpa/9.3_vpa.pdf.

10. Torquetti L, Ferrara P. Corneal asphericity changes after implantation of intrastromal corneal ring segments in keratoconus. J Emmetropia 2010;1:178-81.

11. Y ebra-Pimentel E, González-M éijome J M, Cerviño A, et al. A sfericidad corneal en una poblácion de adultos jóvenes. Implicaciones clínicas. A rch Soc Esp Oftalmol 2004;79:385-92.

12. A mbrosio R J r, A lonso RS, L uz A, Coca V elarde L G. Cornealthickness spatial profile and corneal-volume distribution: 
The Influence of Corneal Volume in Surgical Planning: Insights for a New Parameter for the Ferrara Ring Nomogram

tomographic indices to detect keratoconus. J Cataract R efract Surg 2006;32:1851-59.

13. Pinero D, Alio J, Aleson A, Vergara M E, M iranda M. Corneal volume, pachymetry, and correlation of anterior and posterior corneal shape in subclinical and different stages of clinical keratoconus. J Cataract R efract Surg 2010;36:814-25.

14. Emre S, Doganay S, Y ologlu S. Evaluation of anterior segment parameters in keratoconic eyes measured with Pentacam system. J Cataract R efract Surg 2007;33:1708-12.

15. The Pentacam. The measurement principle. A vailable from: http://www. pentacam.com/sites/messprinzip.php. A ssessed: 2J an 2013.

16. Cervino A, Gonzalez-M eijome J M, Ferrer-Blasco T, GarciaResua C, M ontes-M ico R, Parafita M . D etermination of corneal volume from anterior topography and topographic pachymetry: application to healthy and keratoconic eyes. Ophthalmic Physiol Opt 2009;29(6):652-60.

17. Torquetti L, Ferrara G, Ferrara P. Correlation of anterior segment parameters in keratoconus. IntJ K erat Ect Cor Dis. 2012;1(2)8791.

18. M annion LS, Tromans C, O'D onnell C. Reduction in corneal volume with severity of keratoconus. Curr Eye Res 2011;36(6):522-27.

19. Tu KL, Sebastian RT, Owen M, Batterbury M, Kaye SB. Quantification of the surgically induced refractive effect of intrastromal corneal ring segments in keratoconus with standardized incision site and segment size. J Cataract R efract Surg 20110 ct;37(10):1865-70.

\section{ABOUT THE AUTHORS}

\section{Leonardo Torquetti}

Lecturer, D epartment of Cornea, Paulo Ferrara Eye Clinic, M G, B razil

Correspondence Address: Contorno A ve. 4747, Suite 615 Lifecenter, Funcionários, B elo Horizonte, M G-30110-031, B razil Phone: 553132860836, e-mail: leonardotorqueti@yahoo.com.br leotorquetti@gmail.com

\section{Guilherme Ferrara}

Lecturer, D epartment of Cornea, Paulo Ferrara Eye Clinic, M G, B razil

\section{Wagner Gomes Dias}

Lecturer, D epartment of Cornea, Hilton Rocha Foundation, M G, B razil

\section{Paulo Ferrara}

Professor, Department of Cornea, Paulo Ferrara EyeC Clinic, M G, B razil 